\title{
The Vertical-Cavity Surface Emitting Laser (VCSEL) and Electrical Access Contribution
}

\author{
Angelique Rissons and Jean-Claude Mollier \\ Institut Superieur de l'Aeronautique et de l'Espace (ISAE), \\ Université de Toulouse \\ France
}

\section{Introduction}

This chapter aims at highlighting the influence of the electrical access of different kinds of Vertical-Cavity Surface-Emitting Laser (VCSEL) (Rissons \& Mollier, 2009).

By presenting an overview of electrical equivalent circuit modeling and characterization for the VCSEL chip, based on the Scattering parameters, an overview of the VCSEL performance is given in order to provide a good knowledge of this device behavior in various operation modes and avoid its inadequate utilization (Rissons et al., 2003), (Perchoux et al., 2008), (Bacou et al., 2009).

Due to the emergence of the short distance optical communication, the VCSELs have become, in the past ten years, a key component of the optical interconnections. The current progress in the VCSEL technology makes this laser diode promising for different application fields and competitive with respect to the Edge-Emitting laser (EEL) and the Light Emitting Diode (LED) (providing the laser performance while keeping the cost effectiveness of the LED)(Koyama, 2006),(Suematsu \& Iga, 2008).

Above all the VCSELs have been designed to fulfill the need of optoelectronic circuit planarization. Since its invention in 1977 by Prof. K. Iga (Iga et al., 1988) and its first commercialization at the end of the 90's, the VCSEL structure finds itself in a state of constant progress. Today, it covers a wide wavelength emission range (from the blue-green band up to the infrared) that enables the usage of these components in various application fields, not only in the short distance digital communications (LAN, Avionic network (Ly et al., 2008), ...) but also in consumer applications (laser printers, laser mice, display systems, etc.).
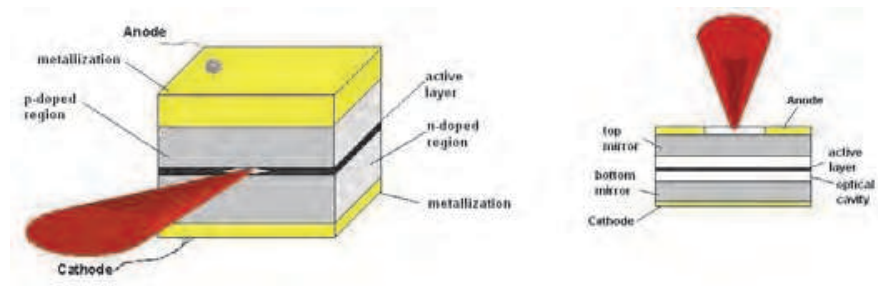

Fig. 1. comparison between Edge Emitting laser (on the left) and VCSEL (on the right) 


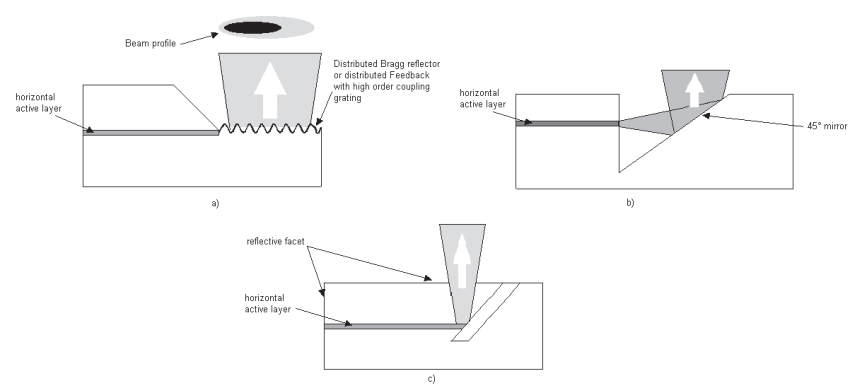

Fig. 2. First structure proposal of Surface Emitting laser

All these applications can be made possible thanks to:

- The vertical emission perpendicular to the semiconductor layers makes easier the integration (1D and 2D Array) according to the electrical packaging constraints.

- By combining the small size and the perpendicular emission, this component responds to the criterion of planarization providing a high integration level.

- The low volume of the active layer (AL) due to the quantum wells (QW) involves a sub-milliamps threshold current and a low electrical power consumption.

- The VCSEL presents a low thermal dependence close to the room temperature.

- The serial fabrication reduces the cost and allows on-wafer testing.

- Due to its cylindrical geometry, the light-beam cross section is circular.

All these reasons have led to the growth of the VCSEL market in a wide application range especially in the integrated optical sub-assemblies of transceivers for short distance optical links. Despite of the numerous advantages of the VCSEL, the available performance has to be improved to face the Distributed-Feedback (DFB) lasers. This improvement could be achieved thanks to the progress in the VCSEL technology, in particular for the structures of 1.3 and $1.55 \mu \mathrm{m}$ (850nm VCSEL technology having matured in the past ten years). Another way to enhance the VCSEL performance and avoid its inadequate utilization, is to have a perfect knowledge of the component behavior in various operating modes. It can be completed by modeling and by measurements, though special attention must be paid to the electrical contribution which hugely modifies the frequency response (O'Brien et al., 2007) (Rissons et al., 2003). Moreover, the current advance in VCSEL technology (intrinsic structure and chip submount) requires a constant review of the model and the characterization before the VCSEL utilization (Bacou et al., 2010). Hence we suggest to dedicate a chapter to the VCSEL technology, that aims at giving an overview of the advances, the physical behavior, and its various structures including the electrical access regarding VCSELs.

\section{VCSEL technology}

\subsection{VCSEL emergence}

The vertical light emitting laser idea emerged between 1975-1977 to satisfy the planarization constraints of the integrated photonics according to the microelectronic technology available then. At first, laser diode researchers proposed structured based on beam deflection (see Fig. 2). After two years of proposals, Prof. K. Iga (Iga et al., 1988) suggested the best solution of 


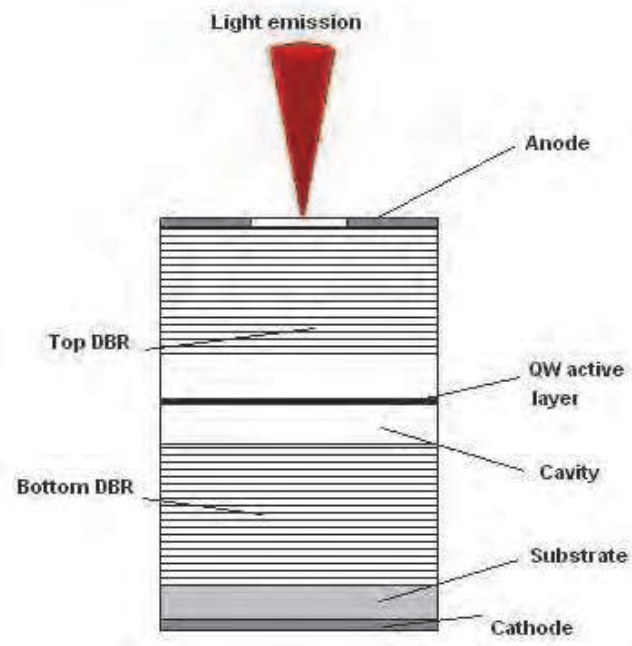

Fig. 3. Vertical-Cavity Surface-Emitting Laser

Vertical-Cavity Surface-Emitting Laser with GaInAsP/InP and AlGaAs/GaAs active region for optical fiber communications, for the optical disks, optical sensing and optical processing. The first goal of Prof. Iga was to grow a monolithic structure in a wafer and test the component before separation. In 1979, the first lasing surface emitting laser (SEL) was obtained with a GaInAsP/InP structure at 77K under pulsed regime. The threshold current was about $900 \mathrm{~mA}$ within 1.3 or $1.55 \mu \mathrm{m}$ wavelength. In 1983, the first lasing at room temperature (RT) under pulsed operation with a GaAs active region was achieved but the threshold current remained higher than the Edge Emitting Laser (EEL)). In spite of the poor VCSEL performance in those days, the progress of the microelectronic technology gave the opportunity to the researcher to improve the VCSEL structure in view of threshold reduction at RT. After a decade of improvement attempts, the first continuous wave (CW) operation at RT was obtained by Iga with a GaAs structure. At the same time, Ibariki (Ibaraki et al., 1989) introduced, into the VCSEL structure, doped Distributed Bragg Reflector (DBR) as mirrors as well for the current injection. Jewell (Jewell et al., 1989) presented the first characterisation of Quantum Wells (QW) GaAs Based Vertical-Cavity Surface Emitting Laser where the DBR and QW introduction is an important breakthrough for the VCSEL technology advance: DBR involves the increase of the reflection coefficient and the QW strongly reduces the threshold current up to few milliamps.

Furthermore, the growth of the VCSEL structure by Molecular Beam Epitaxy (MBE) was a crucial advance toward its performance enhancement. MBE led to a broad-based production (mainly for the AlGaAs/GaAs structure) involving cost effectiveness. Thus, at the beginning of the 90's, we could find the 850nm VCSEL structure presented on Fig. 3, there were still two major drawbacks: the high electrical resistivity of the DBR and the optical confinement through the top DBR. Finding a solution for these problems represented a new challenge in the VCSEL technology.

During 90's, the VCSEL technology research was divided into two branches: on one hand, improving the 850nm VCSEL performance and, on the other hand, designing a 1.3 and a 1.55 $\mu m$ VCSELs. 

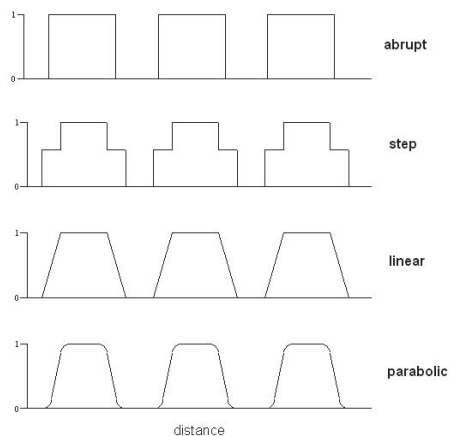

Fig. 4. Various doping profile of $A l_{x} G a_{1-x} A s$

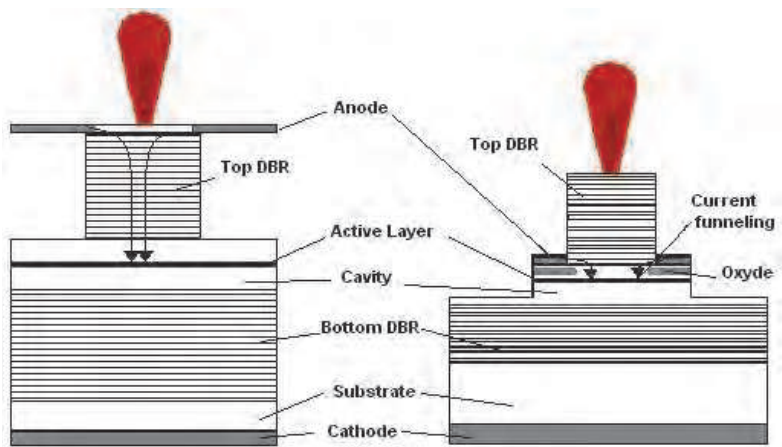

Fig. 5. Mesa structure

\section{$2.2850 \mathrm{~nm}$ VCSEL}

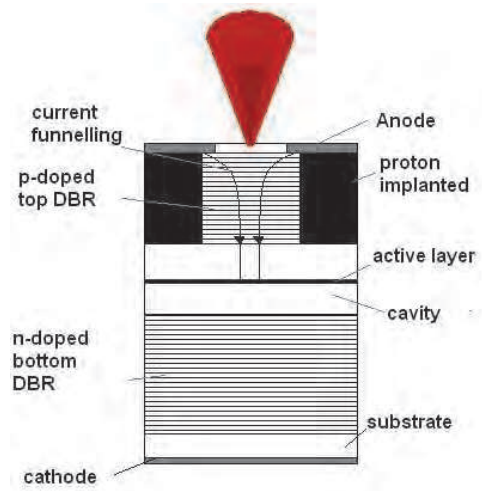

(a)

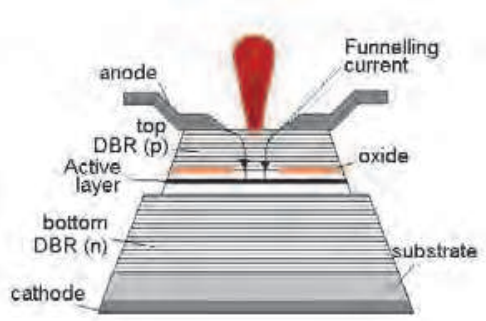

(b)

Fig. 6. (a) Proton implanted VCSEL, (b) Oxide confined VCSEL 
Before attaining the maturity of the 850nm VCSEL technology numerous research works have been carried-out. Firstly, the resistivity of the DBR has been reduced by modifying the doping profile of $A l_{x} G a_{1-x} A s$ (Kopf et al., 1992). Fig. 4 shows different doping profiles. The first VCSEL generations had an abrupt doping profile providing a good reflectivity but a high resistivity $(>100 \Omega)$. By modifying the doping profile at each AlGaAs/GaAs junction, the best compromise between high reflectivity and low resistance has been obtained. The parabolic profile (see fig.4) finally gave the best performance.

The current and photon confinements were other technological bottlenecks. Up to the day, many GaAs-VCSEL structures were proposed. Structures presented on Fig.5 and 6 are the most common. By using a MESA DBR, the optical confinement has been improved. This technology allowed two possibilities for the top electrodes: on the top of the DBR (left side of Fig.5) and closer to the cavity (right side of Fig.5). These structures provided good performance but the technology is in disagreement to the broad-based production. The proton implanted structure presented on the right side of Fig. 6 is the first serial produced VCSEL. The top DBR contains an insulating proton $\left(\mathrm{H}^{+}\right)$layer to limit the current spreading below the top electrode. Nevertheless, this method doesn't reduce enough the injection area to avoid a transverse carrier spreading into the active layer (Zhang \& Petermann, 1994). The main consequence is a multimode transverse emission. Indeed, the coexistence of the optical field and the current funnelling in the same area degrades the VCSEL operation. The oxide confined structure Fig. 6 provides a good compromise between the beam profile and high optical power. Indeed, the diameter of the oxide aperture has an influence on the multimode transverse behavior and the output power. If the oxide aperture diameter is smaller than $5 \mu m$, the VCSEL has a singlemode transverse emission nonetheless the optical power is lower than $1 \mathrm{~mW}$. To obtain a high power VCSEL (about $40 \mathrm{~mW}$ ), the diameter of the oxide aperture has to be wider $(25 \mu \mathrm{m})$ but the beam profile is strongly multimode transverse.

Another point to be emphasized for the use of the 850nm VCSEL is the thermal behavior. As in any semiconductor, the carrier number is strongly dependent on the temperature, while involving fluctuations of the optical power, the wavelength and threshold current (Scott et al., 1993). The earmark of the VCSEL is the parabolic threshold current $\left(I_{t h}\right)$ evolution close to a temperature characteristic. If this characteristic of temperature is close to the ambiant, it has the advantage of avoiding a thermal control for its applications. However the thermal behavior degrades the carrier confinement due to the Joule effect through the DBRs and modifies the refractive index of the DBR. These phenomena are responsible for the multimode transverse emission and strong spatial hole burning.

By knowing these drawbacks, it is possible to consider the VCSEL as a median component between good laser diodes and LED. Its low cost had allowed its emergence into the short distance communication applications to increase the bit rate while keeping cost effectiveness.

\subsection{3 and $1.55 \mu m$ VCSEL}

The emergence of the 1.3 and $1.55 \mu \mathrm{m}$ VCSELs was quite different than the $850 \mathrm{~nm}$ ones. In fact, the telecom wavelength laser market was widely filled by the DFB lasers whose performances are well adapted to the telecom market. Bringing into the market, the LW-VCSEL, the following assets have to be kept versus the DFB: high integration level and cost reduction with relatively good performance. By considering the numerous bottlenecks of the LW-VCSEL technology, it takes up a challenge. The first $1.3 \mu \mathrm{m} \mathrm{CW}$ operation was demonstrated by Iga (Baba et al., 1993),(Soda et al., 1983) in 1993 with an InGaAs/InP based active layer at 77K. The upper mirror was constituted by 8.5 pairs of p-doped $\mathrm{MgO}-\mathrm{Si}$ material with $\mathrm{Au} / \mathrm{Ni} / \mathrm{Au}$ 
layers at the top and 6 pairs of $\mathrm{n}$-doped $\mathrm{SiO} / \mathrm{Si}$ materials at the bottom. The materials given a 1.3 and $1.5 \mu \mathrm{m}$ wavelength are not compatible with a monolithic growth. To provide a wavelength emission within 1.1 - $1.6 \mu \mathrm{m}$ range, the most suitable semiconductor compound is InGaAsP/InP. Even if the wavelength's range is easy to reach, the InGaAsP/InP are not well optimized for the DBR (Shau et al., 2004). Only 12-15 AlAs/AlGaAs pairs are needed to fabricate a DBR with $99 \%$ reflectivity. By taking into account a low refractive index difference (0.3) between $\mathrm{InP} / \mathrm{InGaAsP}$ layer pairs, more than 40 pairs are required to have 99\% reflectivity. The thickness of DBRs has strong consequences on the VCSEL interest, not only in terms of integration but also in terms of heat sinking. In other hand, AlAs/AlGaAs DBR couldn't be grown on InP substrate due to a lattice mismatch. The problem encountered with the DBR utilization has a strong impact into the LW VCSEL technology. In 1997, Salet et Al. (Salet et al., 1997) demonstrated a pulsed RT operation of single-mode InGaAs/InP VCSEL at $1277 \mathrm{~nm}$. The structure was composed by a bottom n-doped InGaAsP/InP DBR (50 pairs) with $99.5 \%$ reflectivity and a top p-doped $\mathrm{SiO}_{2}$ : Si reflector. The threshold current at $300 \mathrm{~K}$ was $500 \mathrm{~mA}$. For each kind of VCSEL, the vertical common path of carrier and photon

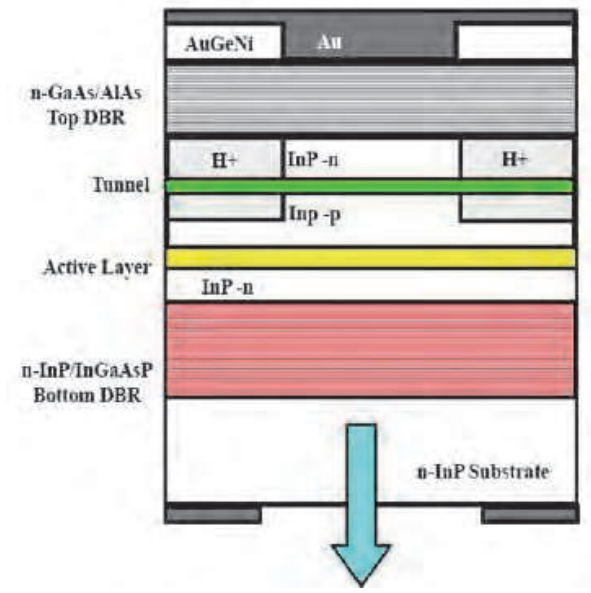

Fig. 7. $1.55 \mu \mathrm{m}$ VCSEL with tunnel junction ((Boucart et al., 1999)

flow has a strong influence on the multimode transverse emission, this unwanted behavior is linked with thermal problems. One of the solutions to segregate the carrier and photon paths was brought by the tunnel junction introduction into the structure. The tunnel junction was discoverd by L. Esaki in 1951 (Esaki, 1974). This junction is composed by two highly doped layers: $n^{++}=p^{++}=1-2 \cdot 10^{19} \mathrm{~cm}^{-3}$. In the case of LW-VCSEL, the tunnel junction acts as a hole generator. With a reverse bias, the electron tunnelling between the valence and the conduction band involves a wide hole population. The tunnel junction has to be localised just above the active layer. Moreover it presents numerous advantages such as the reduction of the intra valence band absorption due to P doping, the threshold current reduction by improving the carrier mobility, the optical confinement. So the tunnel junction is an important technological breakthrough in the LW VCSEL technology. Today, all LW VCSEL include a tunnel junction. In 1999,Boucart et al.(Boucart et al., 1999) demonstrated a RT CW operation of a $1.55 \mu \mathrm{m}$ VCSEL consisting in a tunnel junction and a metamorphic mirror (Fig. 7). A 
metamorphic mirror is a GaAs DBR directly grown on the InP active layer. The threshold current of this structure was $11 \mathrm{~mA}$.

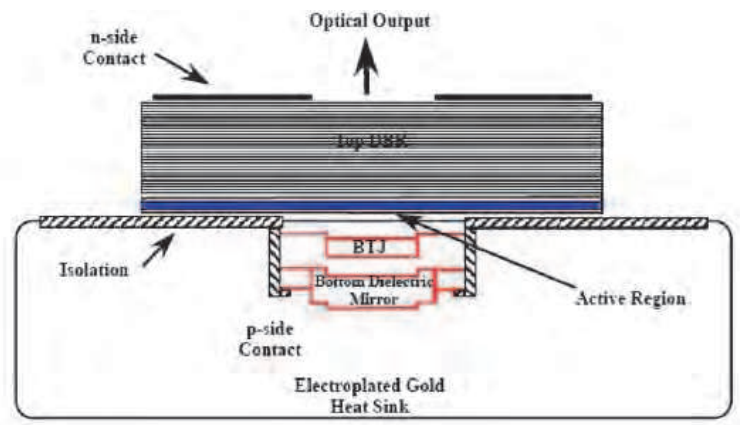

Fig. 8. $1.55 \mu \mathrm{m}$ Vertilas structure

At the same time, Vertilas (Ortsiefer et al., 2000) presented a variation of the Boucart's structure with a bottom dielectric mirror as shown by Fig. 8 . The dielectric mirror provides a $99.75 \%$ reflectivity with only 2.5 pairs of $\mathrm{CaF}_{2} / a-\mathrm{Si}$. Today this kind of structure are commercialised by Vertilas. The performance of these VCSELs, that are in current progress, make them very competitive in the $1.55 \mu m$ VCSEL market.

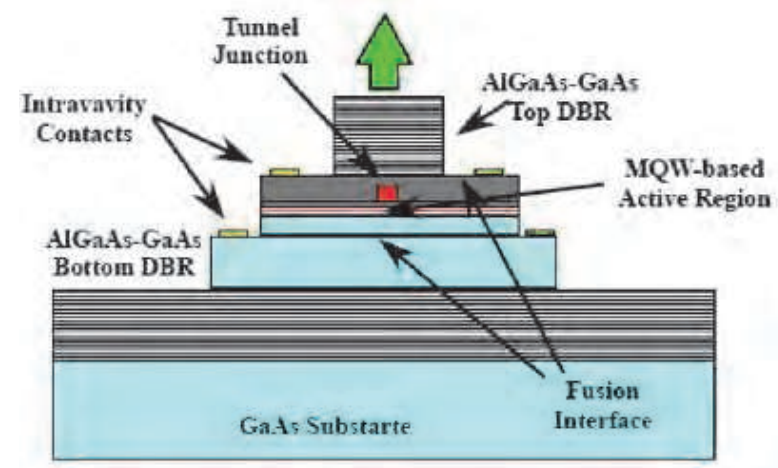

Fig. 9. Wafer fused BeamExpress VCSEL

Another technological breakthrough was the wafer bonding (or wafer fusion) technique. Wafer fusion has been developed by University of California Santa Barbara in 1995 (Babic et al., 1995). Chemical bonds are directly achieved between two materials without an intermediate layer at the heterointerface. A variant of the wafer fusion technique has been demonstrated by Kapon et al. (Syrbu et al., 2004) in order to apply the "localised wafer fusion" to a serial production. This process was developed and patented at Ecole Polytechnique Fédérale de Lausanne (EPFL) where the BeamExpress spin-off emerged. Fig.9 shows the $1.55 \mu \mathrm{m}$ BeamExpress structure. Besides the originality of the localised wafer fusion technique, the carrier injection is also improved by using a double intracavity contact avoiding a current flow through the DBR. Thus a singlemode transverse emission is reached. Today, BeamExpress leads the market in terms of optical power: $6.5 \mathrm{~mW}$ at $1.3 \mu \mathrm{m}$ and $4.5 \mathrm{~mW}$ 
at $1.5 \mu m$ (Kapon et al., 2009).

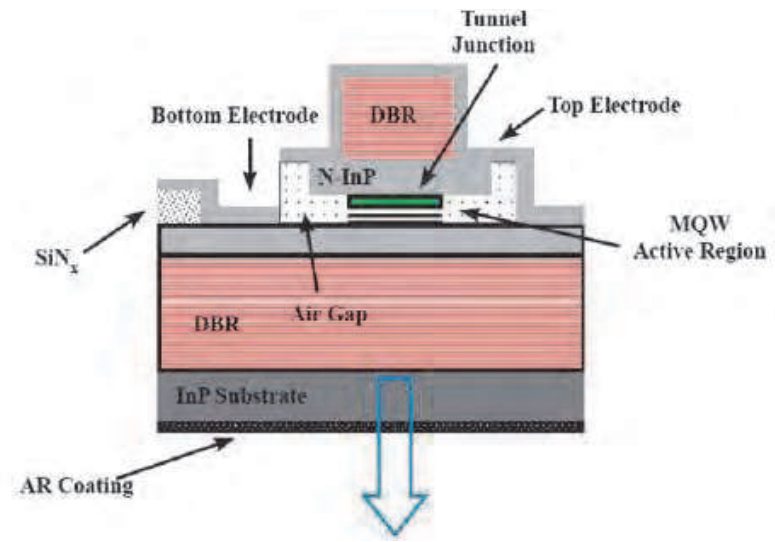

Fig. 10. Monilithic structure of Raycan VCSEL

In 2002, Raycan, a spin-off supported by the Korean Government, launched a project of monolithic long-wavelength VCSEL. They attempted to monolithically grow InAlGaAs DBR and InGaAs-based quantum well active layer on an InP substrate. This technique was unconsidered before because $99 \%$ reflectivity of an InAlGaAs-based DBR required more than 40 pairs. Raycan employed the metal-Organic Chemical Vapour Deposition (MOCVD) technique to fabricate the longwavelength VCSEL. For 1.55 $\mu \mathrm{m}$ VCSELs, the top and bottom DBR were grown as 28 and 38 pairs of undoped InAlGaAs/InAlAs layers. And for the $1.3 \mu \mathrm{m}$ VCSELs, the top and bottom DBRs consisted of 33 and 50 layers respectively. The $0.5 \lambda$ thick active region consists of seven pairs of strain-compensated InAlGaAs QW. The lower pair number of the top DBR was compensated by using an InAlGaAs phase-matching layer and a Au metal layer. Fig. 10 presents the structure of $1.55 \mu \mathrm{m}$ Raycan VCSEL. Reliable structure (Rhew et al., 2009) are being commercialised since 2004.

\subsection{Electrical access topology}

Up to this point, we have presented the main VCSEL structures without taking into account the electrical access topology. Knowing the VCSEL structure facilitates the understanding of the mecanism of electron-photons but it is insufficient to foresee the VCSEL behavior under modulation. As for the edge emitting laser (Tucker \& Pope, 1983), the VCSEL modulation response is affected by parastic elements due to the connection with the input electrical source. The electrical access is the most influential in the VCSEL array configuration. Despite of its high integration level the VCSEL technology, the electrical connection ensuring the driving is not immediate and requires an optimization in order to match the VCSEL with its driving circuit. Up to the day, the VCSEL are shipped into various packages. Each package is available for an associated frequency application range. The increases in frequency involve a specific electrical access to limit parasitics effects. But as it will be shown, even for the VCSEL chip, the electrical access modifies the VCSEL frequency response. Before continuing, let us dwell on the different chip types and the packages.

The VCSEL chip topology presents top and bottom electrodes. According to the intrinsic structure, we could have two kind of VCSELs: the "top-emitting VCSELs" where the signal 
is brought through the top electrode and the ground linked to the bottom electrode, on the other hand, the "bottom-emitting VCSELs" have the ground contact on the top and the signal contact on the bottom. Thus, the topology of the chip will depend on the top and bottom emission.

- Microstrip electrical access

A great deal of VCSEL arrays are manufactured with a signal access on the top and a bottom common ground as we can see on Fig.11.

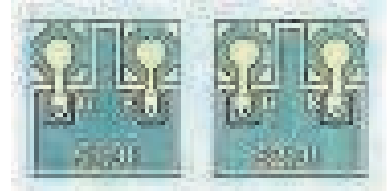

Fig. 11. Microstrip access

This topology is perfectly adapted to the top emitting VCSEL. It allows to share the ground face of each VCSEL of an array. The signal, on the top, is achieved by a microstrip line matched to each VCSEL of the array. Such a structure has the advantage of reducing the spacing between each VCSEL of an array. In order to test the VCSEL, it is necessary to mount the array on a TO package or on a submount with etched strip lines.

Due to its technological simplicity, TO package is a common packaging for a single
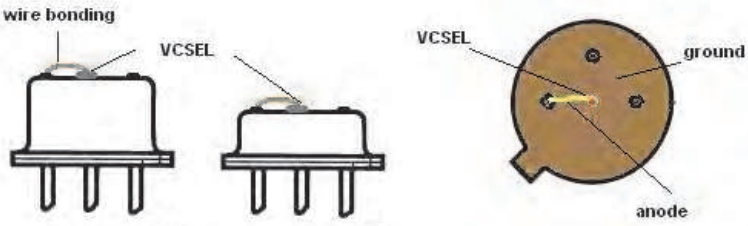

TO 18

TO 46

Fig. 12. TO package

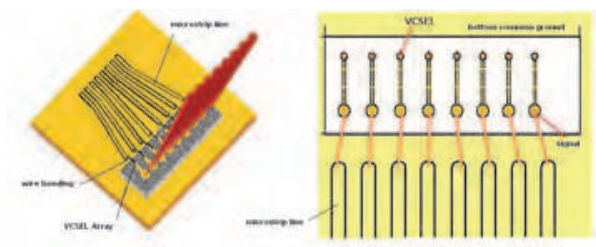

Fig. 13. VCSEL array submount

VCSEL and sometimes for VCSEL array. As shown in Fig.12, the VCSEL is fused on the top of the TO package. The ground contact, on the bottom, is carried out through the welding, that is to say, the ground is linked to the metal can. The signal contact is provided by a wire bonding between the VCSEL strip line and a pin isolated to the metal can. Many VCSELs are available on TO package with connector, lens caps or pigtailed. The utilisation of a TO packaged VCSEL is easy and allows to do many characterisations such as optical 
power versus bias current, optical spectrum, linewidth and Relative Intensity Noise (RIN). Unfortunately it is not well adapted for the high frequency application. Actually, the TO packaging presents a frequency limitation between 2 an $4 \mathrm{GHz}$, often below VCSEL cut-off frequency. That is why the utilisation of a TO packaged VCSEL is inadvisable for high frequency modulation. Reliable mathemathical extraction procedures are available for the frequency response study (Cartledge \& Srinivasan, 1997) but, in a goal of integration in an optical sub-assembly, the modulation frequency or the bit rate would not be optimized.

In the case of a VCSEL array, the TO package is not well adapted. Thus it is necessary to set the array on a submount with etched strip lines. As it is presented by Fig. 13, the electrical connection with the array is realized by using wire bondings.

The common ground of the VCSEL array is linked with the ground of the etched strip lines. However, this submount involves parasitic effects clearly visible under modulation operation (Rissons \& Mollier, 2009). A coupling between adjacent VCSEL is observable: when one VCSEL is modulated, the neighbouring VCSEL lase without any injection (we will return to this point in a further section). This coupling increase with the frequency but according to these drawback, the microstrip line electrical access is not the best configuration for the frequency modulation.

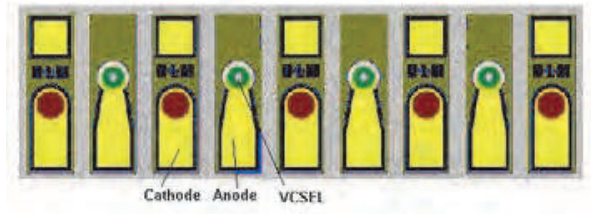

Fig. 14. Coplanar electrical access VCSEL array

\section{- Coplanar electrical access}

Another available VCSEL array chip presents a coplanar access. This topology is in a good agreement with the planarization. As Fig.14 shown, not only the anode but also the cathode (which is rised by via-hole) are on the top of the chip. This topology have the advantage to minimize the length of the electrical access and reduce the parasitics phenomena. Moreover, the coplanar access allows an impedance matching to limit the electrical reflection on the VCSEL input. This configuration is ideal for the RF test because the RF probe could be placed closer to the chip. Regarding to the VCSEL array, no coupling phenomema between adjacent VCSEL have been observed. Finally the integration is easyer than the microstrip access due to the ground on the top. Nevertheless, wire bondings are required to connect the VCSEL array with its driver.

\section{- Bottom-emitting VCSEL chip}

The electrical access toplogy previously presented is not adapted to the bottom-emitting VCSEL. The flip-chip bonding is required for the electrical contact. This technique has the advantage to be suitable for the integration on a CMOS circuit. Several VCSEL manufacturers provide this kind of chip. Fig. 15 shows the topology of a Raycan VCSEL chip. In counterpart, the RF testing is difficult because the bottom emission implies the impossibility of optical power collection. 


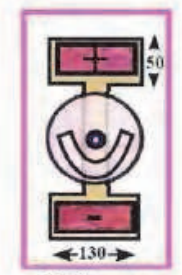

Units: $\mu \mathrm{m}$

Fig. 15. Bottom-emitting Raycan VCSEL chip

\section{Optoelectronic model: rate-equations and equivalent circuit model}

This section aims at presenting a complete model of VCSEL in order to be able to simulate the VCSEL behavior before its implementation in an optical sub-assembly. Firstly, the rate equations are defined according to the VCSEL structure and simplified in compliance with the operating mode. The steady state model and characterization through the light current model is developed. Secondly, we will be interested in the dynamic behavior of the VCSEL. This approach is based on the comparison between the rate equations and an electrical equivalent circuit to obtain the relationships between intrinsic parameters and equivalent circuit elements (Tucker \& Pope, 1983),(Bacou et al., 2010). The electrical equivalent circuit approach consists in describing the physical phenomena occurring into the VCSEL structure by resistive, inductive and capacitive elements. The behavioral electrical equivalent circuit is cascaded with the electrical access circuit according to each submount.

\subsection{VCSEL rate equations}

As for each laser diodes, the electron-photon exchanges into the VCSEL are modeled by a set of coupling rate equations. These equations relate the physical mecanisme inside the VCSEL structure, thus each approximation has to take into account the variant of each VCSEL.

The carrier rate equation is the difference between the carrier injection and the carrier recombinations. The photon rate equation is the difference between the generated photons participated to the stimulated emission and the lost photons. These equations can be written as the following form:

$$
\begin{gathered}
\frac{d N}{d t}=\frac{\eta_{i} \cdot I}{q \cdot N_{w}}-\left(A+B \cdot N+C \cdot N^{2}\right) N-G \cdot S+F_{N}(t) \\
\frac{d S}{d t}=\Gamma \cdot \beta \cdot B \cdot N^{2}+N_{w} \cdot G \cdot S-\frac{S}{\tau_{S}}+F_{S}(t)
\end{gathered}
$$

Where:

- $N$ is the carrier number in one QW, $S$ is the photon number in the cavity.

- $N_{w}$ is the QW number. $\eta_{i}$ is the internal quantum efficiency. $I$ is the injected current. So $\frac{\eta_{i} \cdot I}{q \cdot N_{w}}$ represents the population injection into each QW.

- $A$ is the non-radiative recombinations (by recombinant center), $B$ is the bimolecular recombination (representing the random spontaneous emission), $C$ is the Auger recombination coefficient which can be neglected for the sub-micron emitting wavelength. We can consider $A+B \cdot N+C \cdot N^{2}=\tau_{n}^{-1}$ where $\tau_{n}$ is the carrier lifetime which could be taken as a constant according to the laser operation mode. 
- $G$ is the modal gain. It depends to the carrier and photon number through the relationship $G(N, S)=g_{0} \cdot \frac{N-N_{t r}}{1+\epsilon S}$ where $N_{t r}$ is the transparency electron number, $\epsilon$ is the gain compression factor. The modal gain coefficient $g_{0}$ is expressed as $g_{0}=v_{g r} \cdot \Gamma \cdot \frac{a}{V_{a c t}}$ where $a$ is the differential gain coefficient, $V_{a c t}$ is the active layer volume, $v_{g r}$ the group velocity and $\Gamma$ is the confinement factor.

- The term $\Gamma \cdot \beta \cdot B \cdot N^{2}$ corresponds to the spontaneous emission contributing to the lasing mode. $\beta$, the spontaneous emission coefficient, relates the portion of the spontaneous emission which will be amplified.

- $\tau_{S}$ is the photon lifetime into the cavity. It is linked to the loss by the relationship $\tau_{S}^{-1}=$ $v_{g r} \cdot\left(\alpha_{i}+\alpha_{m}\right), \alpha_{i}$ represents the internal losses and $\alpha_{m}$, the mirror losses.

These equations are adapted to a QW laser through the $\eta_{i}$ value and the presence of $N_{w}$. The confinement factor takes into account the vertical light emission and the DBR contribution. Moreover the values of each intrinsic parameters depend on the VCSEL structure.

The two last term $F_{N}(t)$ and $F_{S}(t)$ have to be taken in part. In fact, $F_{N}(t)$ and $F_{S}(t)$ are the carrier and photon Langevin functions respectively, representing the carrier and electron fluctuations. These fluctuations are due to the stochastic evolution of $N$ and $S$ associated to the noise generation. Indeed, the operation of the laser diode is affected by several noise sources whose influence varies according to the different regimes. For targeted applications, the preponderant noise source is the spontaneous emission. The randomness of the spontaneous emission generates amplitude and phase fluctuations of the total optical field. Moreover, these photons which are produced in the laser cavity follow the feedback of the stimulated photons and interact with them. By taking into account the wave-corpuscule duality of the light, a quantum approach is well suited to describe the emission noise generation including the photon-electron interaction: each state of photon or electron is associated to a noise pulse. For the purposes of noise generation quantification, recombination and absorption rates in the cavity allow the utilization of the electron and photon Langevin forces to give a mathematical representation of the optical emission noise.

To complete the VCSEL modeling, rate equations have to be solved according to each operation mode.

\subsubsection{Steady state resolution}

The first step of the rate equation resolution considers the case of the steady state. This resolution aims at to extract the relationship of the threshold current, threshold carrier number, and current/photons relations above threshold. It also allows to valid which approximation degrees are reliable.

When the steady state is reached, the rate equations are equal to 0 such as:

$$
\begin{gathered}
0=\frac{\eta_{i} \cdot I}{q \cdot N_{w}}-\left(A+B \cdot N+C \cdot N^{2}\right) N-G \cdot S \\
0=\Gamma \cdot \beta \cdot B \cdot N^{2}+N_{w} \cdot G \cdot S-\frac{S}{\tau_{S}}
\end{gathered}
$$

Fig.16 represents the carrier and photon evolution versus the bias current where the red straight line corresponds to an asymptotic representation and the dotted line correponds to a physical representation. So we will study both cases begining by an asymptotic resolution involving that the spontaneaous emission $\Gamma \cdot \beta \cdot B \cdot N^{2}$ and the gain compression $\epsilon \cdot S$ are neglected. 

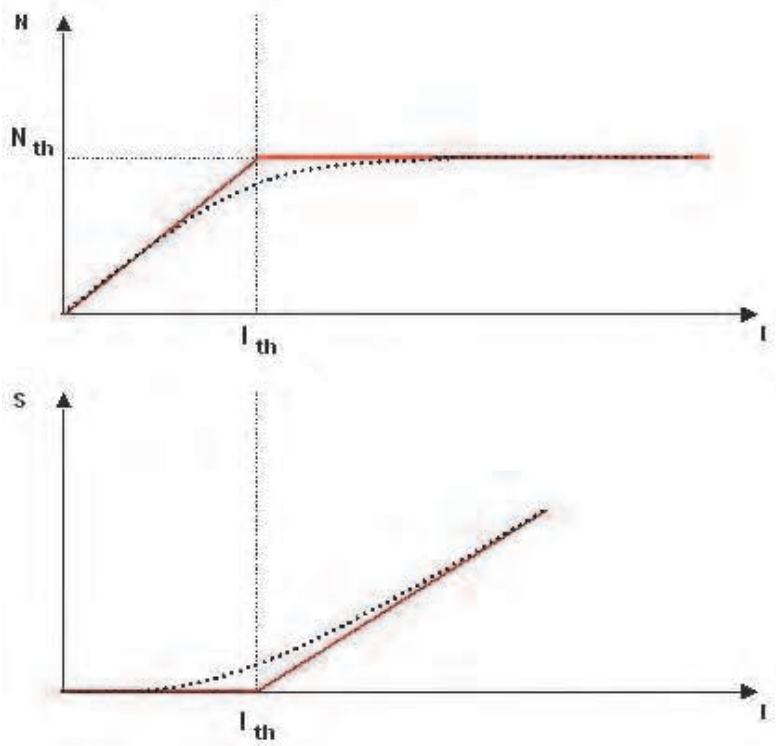

Fig. 16. Evolution of carrier number $(N)$ and photon number $S$ versus the bias current $I$

- Below threshold: $I<I_{t h}, N<N_{t h}$ et $S \approx 0$, according to Fig.16

From the equation 3 , we can extract:

$$
N=\frac{\tau_{n} \cdot \eta_{i} \cdot I}{q \cdot N_{w}}, \forall N \leq N_{t h}
$$

- Above threshold: $S>0$ and $I>I_{t h}$

From the equation 4 , we obtain:

$$
N_{w} \cdot g_{0}\left(N-N_{t r}\right)=\frac{1}{\tau_{S}}
$$

Involving:

$$
N=N_{t r}+\frac{1}{N_{w} \cdot \tau_{S} \cdot g_{0}}=c t e=N_{S}
$$

Which is equivalent to say: while the simulated emission occures, the carrier number stops to increase linearly with the current because the carrier consumption is compensated by the injected current. Thus, we can consider that the carrier number is constant above the threshold.

From the equations 5 and 7 , the following relationship could be extracted:

$$
I_{t h}=\frac{q \cdot N_{w} \cdot N_{t h}}{\eta_{i} \cdot \tau_{N}}
$$

Thus from the equations 3, 6 and 8 , we obtain:

$$
S=\frac{\tau_{S} \cdot \eta_{i}}{q \cdot N_{w}}\left(I-I_{t h}\right)
$$


Then for $I>I_{t h}$, the photon number linearly increases with the current which verifies the well-known relationship versus the optical power such as:

$$
P_{o p t}=v_{g r} \cdot \alpha_{m} \cdot h \cdot v \cdot S=\eta_{d} \cdot \frac{h \cdot v}{q \cdot N_{w}}\left(I-I_{t h}\right)
$$

where $\eta_{d}$ is the slope efficiency such as: $\eta_{d}=\eta_{i} \frac{\alpha_{m}}{\alpha_{i}+\alpha_{m}}$.

By considering the spontaneaous emission contribution, we can express the real N(I) and S(I) curves close to the threshold according to the dotted line on Fig.16. Henceforth in equation 4, the term $\Gamma \cdot \beta \cdot B \cdot N$ is different from zero. As the following equation is valid:

$$
N_{w} \cdot g_{0}\left(N_{t h}-N_{t r}\right)=\frac{1}{\tau_{S}}
$$

The photon rate equation becomes:

$$
0=\Gamma \cdot \beta \cdot B \cdot N^{2}+g_{0} \cdot N_{w} \cdot\left(N-N_{t h}\right) \cdot S
$$

So for each injected current, the photon number is not negligible because of the spontaneous emission. Thus, the amplified spontaneous emission is expressed in the following relationship:

$$
S=\frac{\Gamma \cdot \beta \cdot B \cdot N^{2}}{g_{0} \cdot N_{w} \cdot\left(N-N_{t h}\right)}
$$

By injecting Equation 13 in Equation 3, the relationship between $\mathrm{N}$ and $\mathrm{I}$ is written as:

$$
\frac{\eta_{i} \cdot I}{q \cdot N_{w}}-\frac{N}{\tau_{N}}-\left(N-N_{t r}\right) \frac{\Gamma \cdot \beta \cdot B \cdot N^{2}}{N_{w} \cdot\left(N-N_{t h}\right)}=0
$$

The solution of the $3^{r d}$ equation corresponds to the evolution of the carrier number close to the threshold. According to Fig.16 and while $I$ is close to its threshold value, the spontaneaous emission slows the increase of the carrier number to reach the steady-state.

Now, considering the gain compression, that is to say for high value of photon number involving: $\epsilon \cdot S>1$. The rate equation becomes:

$$
\begin{gathered}
0=\frac{\eta_{i} \cdot I}{q \cdot N_{w}}-\frac{N}{\tau_{N}}-g_{0} \frac{N-N_{t r}}{1+\epsilon \cdot S} \cdot S \\
0=N_{w} \cdot g_{0}\left[\frac{N-N_{t r}}{1+\epsilon \cdot S}-\left(N_{t h}-N_{t r}\right)\right] \cdot S
\end{gathered}
$$

Thus:

$$
\frac{N_{t h}-N_{t r}}{N-N_{t r}}=\frac{1}{1+\epsilon \cdot S}
$$

and

$$
S=\frac{N_{w} \cdot g_{0} \cdot \tau_{S}}{\epsilon}\left(N-N_{t h}\right)
$$

By injecting Equations 17 and 18 in Equation 15, we obtain:

$$
\frac{\eta_{i} \cdot I}{q \cdot N_{w}}-\frac{N}{\tau_{N}}-\frac{g_{0}}{\epsilon}\left(N-N_{t h}\right)=0
$$


In fact the overflow of the carrier population versus the steady-state value causes an overflow of photon responsible to the gain compression. We can note $\delta N$ the carrier overflow, so that: $N=N_{t h}+\Delta N$.

From the equation 19, we obtain:

$$
\delta N=\frac{\eta_{i}}{q \cdot N_{w}} \frac{\tau_{N} \cdot \epsilon}{\tau_{N} \cdot g_{0}+\epsilon}\left(I-I_{t h}\right)=0
$$

If $\tau_{N}=10^{-9} s, \epsilon=10^{-7}, g_{0}=10^{4} \mathrm{~s}^{-1}, I-I_{t h}=10^{-3} \mathrm{~A}$ and $\eta_{i}=10^{-2}, \delta \mathrm{N}$ will be approximatively equal to $10^{3}$ which is negligible against $N_{t h}$. Hence, we can admit the validity of the asymptotic approximation.

\subsubsection{Small-signal linearization}

Now, the VCSEL is modulated by the superimposition of the bias current $I_{0}$ and a sinusoidal current $\Delta I(t)$ so that $\Delta I(t)<<I_{0}$. Due to the small-signal approximation, $N(t)$ and $S(t)$ follow $I(t)$. Let us now linearizing the rate equations according to these conditions. As $X(t)=$ $X_{0}+\Delta X(t)$ (where $X(t)=I(t), N(t)$ or $\left.S(t)\right), \frac{d X_{0}}{d t}=0$ and $\frac{d X(t)}{d t}=\frac{d \Delta X(t)}{d t}$. By putting $\dot{X}=\frac{d X}{d t}$, we have $\frac{d \delta X}{d t}=\delta \dot{X}$. As $\dot{N}$ depends on $I, N, S$ and $\dot{S}$ on $N$ and $S$, we have:

$$
\begin{gathered}
\Delta \dot{N}=\frac{\partial \dot{N}}{\partial I} \Delta I+\frac{\partial \dot{N}}{\partial N} \Delta N+\frac{\partial \dot{N}}{\partial S} \Delta S \\
\Delta \dot{S}=\frac{\partial \dot{S}}{\partial N} \Delta N+\frac{\partial \dot{S}}{\partial S} \Delta S
\end{gathered}
$$

After linearization, we obtain:

$$
\begin{aligned}
\Delta \dot{N} & =\frac{\eta_{i}}{q \cdot N_{w}} \Delta I-\left(g_{0} \cdot \frac{S_{0}}{1+\epsilon \cdot S_{0}}+A+2 \cdot B \cdot N_{0}+3 \cdot N_{0}^{2}\right) \Delta N+g_{0} \frac{N_{0}-N_{t r}}{\left(1+\epsilon \cdot S_{0}\right)^{2}} \Delta S \\
\Delta \dot{S} & =\left(2 \cdot \Gamma \cdot \beta \cdot B \cdot N_{0}+N_{w} \cdot g_{0} \frac{S_{0}}{1+\epsilon \cdot S_{0}}\right) \Delta N+\left(N_{w} \cdot g_{0} \frac{N_{0}-N_{t r}}{\left(1+\epsilon \cdot S_{0}\right)^{2}}-\frac{1}{\tau_{S}}\right) \Delta S
\end{aligned}
$$

The equation set can be expressed as follows:

$$
\left[\begin{array}{c}
\Delta \dot{N} \\
\Delta \dot{S}
\end{array}\right]=[M]\left[\begin{array}{c}
\Delta N \\
\Delta S
\end{array}\right]+\frac{\eta_{i}}{q \cdot N_{w}}\left[\begin{array}{c}
\Delta I \\
0
\end{array}\right]
$$

$\mathrm{M}$ is a matrix such as:

$$
[M]=\left[\begin{array}{cc}
-\gamma_{N N} & -\gamma_{N S} \\
\gamma_{S N} & -\gamma_{S S}
\end{array}\right]
$$

where:

$$
\begin{gathered}
\gamma_{N N}=A+2 B N_{0}+3 C N_{0}^{2}+\frac{g_{0} \cdot S_{0}}{1+\epsilon \cdot S_{0}} \\
\gamma_{N S}=\frac{g_{0}\left(N_{0}-N_{t r}\right)}{\left(1+\epsilon S_{0}\right)^{2}} \\
\gamma_{S N}=2 \Gamma \beta B N_{0}+\frac{N_{w} \cdot g_{0} \cdot S_{0}}{1+\epsilon S_{0}}
\end{gathered}
$$




$$
\gamma_{S S}=\frac{1}{\tau_{S}}-\frac{N_{w} \cdot g_{0}\left(N_{0}-N_{t r}\right)}{\left(1+\epsilon S_{0}\right)^{2}}
$$

By considering the sinusoidal modulation as $\Delta I=I_{m} \cdot e^{j \omega t}, \Delta N=N_{m} \cdot e^{j \omega t}$ and $\Delta S=S_{m} \cdot e^{j \omega t}$, and with $\frac{d}{d t}=j \omega$, the Equation 25 becomes:

$$
\left[\begin{array}{cc}
\gamma_{N N}+j \omega & \gamma_{N S} \\
-\gamma_{S N} & \gamma_{S S}+j \omega
\end{array}\right]\left[\begin{array}{c}
\Delta N \\
\Delta S
\end{array}\right]=\frac{\eta_{i}}{q}\left[\begin{array}{c}
\Delta I \\
0
\end{array}\right]
$$

By using the Cramer rule, we obtain:

$$
\begin{aligned}
& N_{m}=\frac{\eta_{i} I_{m}}{q} \frac{1}{\Delta}\left|\begin{array}{cc}
1 & \gamma_{N S} \\
0 & \gamma_{S S}+j \omega
\end{array}\right| \\
& S_{m}=\frac{\eta_{i} I_{m}}{q} \frac{1}{\Delta}\left|\begin{array}{cr}
\gamma_{N N}+j \omega & 1 \\
-\gamma_{S N} & 0
\end{array}\right|
\end{aligned}
$$

where $\Delta$ is the matrix determinant such as:

$$
\Delta=\gamma_{N N} \gamma_{S S}+\gamma_{N S} \gamma_{S N}-\omega^{2}+j \omega\left(\gamma_{N N}+\gamma_{S S}\right)=\omega_{R}^{2}-\omega^{2}+j \omega \gamma_{R}
$$

$\omega_{R}$ is the resonance frequency such as: $\omega_{R}^{2}=\gamma_{N N} \gamma_{S S}+\gamma_{N S} \gamma_{S N}$

et $\gamma_{R}$ is the damping factor: $\gamma_{R}=\gamma_{N N}+\gamma_{P P}$

Which is conform to a transfer function of a second order system. The resonance frequency is an important parameters in the determination of the VCSEL frequency bandwidth. We will make some approximation according to the small signal regime. By considering a bias current $I_{0}$ above threshold $\left(I>2 \cdot I_{t h}\right)$, in this context, the spontaneous emission and the gain compression can be neglected. Moreover as we are above threshold, the non-radiative and bimolecular recombination are negligible against the stimulated emission. So $\gamma_{N N}, \gamma_{N S}, \gamma_{S N}$ and $\gamma_{S S}$ become:

$$
\begin{gathered}
\gamma_{N N}=g_{0} \cdot S_{0} \\
\gamma_{N S}=g_{0}\left(N_{0}-N_{t r}\right) \\
\gamma_{S N}=N_{w} \cdot g_{0} \cdot S_{0} \\
\gamma_{S S}=\frac{1}{\tau_{S}}-N_{w} \cdot g_{0}\left(N_{0}-N_{t r}\right)
\end{gathered}
$$

Which allow us to determine the resonance frequency as:

$$
f_{R}=\frac{\omega_{R}}{2 \pi}=\frac{1}{2 \cdot \pi} \cdot \sqrt{\frac{g_{0} \cdot S}{\tau_{S}}}=\sqrt{\frac{g_{0} \cdot \eta_{i}}{q \cdot N_{w}}\left(I-I_{t h}\right)}
$$

This relationship is only reliable above threshold. If the bias current had been close to the threshold, the approximation couldn't be suitable. Moreover, this resonance frequency doesn't take into account the electrical access. We will see how to take into account the electrical access in a model. 


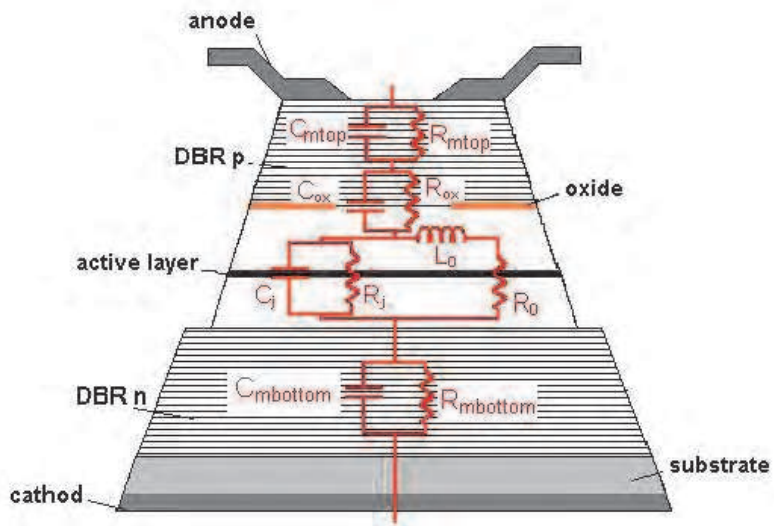

Fig. 17. Behavioral equivalent circuit of a 850nm VCSEL

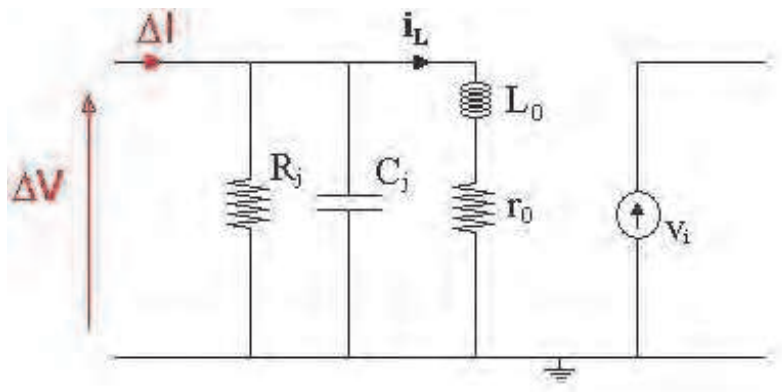

Fig. 18. Small-signal driving of a VCSEL cavity equivelent circuit

\subsection{Behavioral equivalent circuit}

This approach consists in describing the physical phenomena occuring into the VCSEL structure by an equivalent circuit as shown in Fig.17 for an oxide-confined VCSEL emitting at $850 \mathrm{~nm}$. This concept is frequently used in EEL since it has been proposed by R.S Tucker (Tucker \& Pope, 1983)in the 80's. As the electronic funneling through the VCSEL structure is different than the EEL one, the electrical equivalent circuit needs to be adapted by including the multi-quantum well $(\mathrm{QW})$, as well as the active layer represented by a RLC resonant circuit (17): $R_{j}$ and $C_{j}$ are associated to the carrier exchanges, $L_{0}$ and $R_{0}$ are the photon storage and resonance damping respectively. For the 850nm VCSEL, the doped DBR, which is a stack of doped heterojunctions, is equivalent to distributed RC cells: $R_{m t o p}$ and $C_{m t o p}$ for the top DBR, $R_{\text {mbottom }}$ and $C_{\text {mbottom }}$ for the bottom one. As the current confinement is performed by an oxide aperture, a $R_{o x} C_{o x}$ cell is added to the circuit (Brusenbach et al., 1993). This behavioral electrical equivalent circuit can be directly cascaded with the electrical access according to each submount.

By using the linearized rate equation and the Kirchhoff equation of the circuit, relationships between intrinsic parameters and circuit element can be achieved.

As the rate equation considers the carrier number in each QW and the photon number into the cavity, the Kirchhoff equations are limited to the equivalent circuit of the active region. The equation of the cavity equivalent circuit are expressed according to the convention given 
by Fig.18 where $\Delta V$ and $\Delta I$ are the input voltage and current respectively, and $i_{L}$ is the photonic current related to the photon flow variation.

Then, we obtain the following equations:

$$
\begin{gathered}
\frac{d \Delta V}{d t}=\frac{\Delta I}{C_{j}}-\frac{\Delta V}{R_{j} \cdot C_{j}}-\frac{i_{L}}{C_{j}} \\
\frac{d i_{L}}{d t}=\frac{\Delta V}{L_{0}}-\frac{R_{0} \cdot i_{L}}{L_{0}}
\end{gathered}
$$

With these equations the relationship with the instrinsic parameters can be etablished.

\subsection{Relationship between rate equation and equivalent circuit}

To write the relationships between VCSEL intrinsic parameters and the circuit elements, Equations 23,24,40, 41 have to be compared by using the well-known relationship derived from the voltage-current characteristic of a junction diode:

$$
\frac{\Delta V}{V_{T}}=\frac{\Delta I}{I_{0}}=\frac{\Delta N}{N_{0}}
$$

Where $V_{T}$ is assumed to be a constant according to the semiconductor material: $V_{T}=\frac{m \cdot k \cdot T}{q}$. Thus:

$$
\frac{\Delta N}{d t}=\frac{N_{0}}{V_{T}} \cdot \frac{d \Delta V}{d t}
$$

That allow us to obtain directly:

$$
\begin{gathered}
C_{j}=\frac{N_{0} \cdot q \cdot N_{w}}{V_{T} \cdot \eta_{i}} \\
R_{j}=\left[\frac{N_{0} \cdot q \cdot N_{w}}{V_{T} \cdot \eta_{i}} \cdot\left(A+2 \cdot B \cdot N_{0}+3 \cdot C \cdot N_{0}^{2}+\frac{g_{0} \cdot S_{0}}{1+\epsilon \cdot S}\right)\right]^{-1}
\end{gathered}
$$

The relationship between the current $i_{L}$ and the photon fluctuations $\Delta S$ is:

$$
g_{0} \cdot \frac{N_{0}-N_{t r}}{\left(1+\epsilon \cdot S_{0}\right)^{2}}=\frac{N_{0}}{V_{T} \cdot C_{j}} i_{L}
$$

By injecting Equation 46 into Equation 24, we obtain:

$$
\begin{gathered}
L_{0}=\frac{1}{C_{j}} \cdot \frac{(1+\epsilon \cdot S)^{2}}{g_{o}\left(N_{0}-N_{t r}\right) \cdot\left(2 \cdot \Gamma \cdot \beta \cdot B \cdot N_{0}+\frac{g_{0} \cdot S_{0}}{1+\epsilon \cdot S_{0}}\right)} \\
\frac{R_{0}}{L_{0}}=\frac{1}{\tau_{S}}-g_{0} \frac{N_{0}-N_{t r}}{(1+\epsilon \cdot S 0)^{2}}
\end{gathered}
$$

From the convention of Fig. 18, the resonance frequency can be expressed as follow:

$$
f_{R}=\frac{\omega_{R}}{2 \cdot \pi}=\frac{1}{2 \cdot \pi} \cdot \sqrt{\frac{1}{L_{0} \cdot C_{j}}+\frac{R_{0}}{L_{0}} \cdot \frac{1}{R_{j} \cdot C_{j}}}
$$

So by modulating the VCSEL in small signal conditions, some intrinsic parameters can be extracted. The task would be easy if the contribution of the electrical access were to be 
negligeable. In practice, the strong contribution of the electrical access in the frequency response hide the VCSEL response. That's why the equivalent circuit approach is an excellent tool to cascade the electrical access and the laser diode. We will see how to extract the instrinsic resonance frequency and some intrinsic parameters.

\section{VCSEL chip characterisation}

As the frequency response of a laser diode is in the microwave domain, the modulation required a particular care. Indeed, the driving signal could be consider as a simple current-voltage couple but as an electromagnetic field that propagate as a standing-wave. thus, the connection between the VCSEL and the transmission line being achieved by a transmission line, an impedance matching between the driver and the VCSEL is required yet it is not the case of many available VCSEL. This impedance mismatch involves a high reflection on the VCSEL input, that is to say the modulation signal is not totally transmitted to the VCSEL, and the energy of the electromagnetic field radiates nearby the transmission line. To characterize the VCSEL chip by taking into account the electrical access, the scattering (S) parameters measurement with a vector network analyzer is the most suitable. This method allows not only to measure the frequency response of the VCSEL but also to extract the electrical access effect.

\subsection{S-parameters measurement}

In microwave circuit, the voltage, current and impedance cannot be measured in a direct manner. The measurable quantities are the amplitude and phase of the transmitted wave as compared with those incident wave. These quantities are defined as the Scaterring Matrix (S-Matrix). For a two port system, the S-matrix is written as follow:

$$
[S]=\left[\begin{array}{ll}
S_{11} & S_{12} \\
S_{21} & S_{22}
\end{array}\right]
$$

Where:

- $S_{11}$ is the reflection coefficient on the input (1-port)

- $S_{22}$ is the reflection coefficient on the output (2-port)

- $S_{21}$ is the transmission coefficient through the system from the 1-port to the 2-port

- $S_{12}$ is the transmission through the system from the 2-port to the 1-port

For a laser diode, the $\mathrm{S}$ matrix becomes:

$$
[S]=\left[\begin{array}{ll}
S_{11} & 0 \\
S_{21} & 1
\end{array}\right]
$$

$S_{12}=0, S_{22}=1$ if no optical feed-back into laser cavity is considered. So that for the microwave-photonic S-Matrix measurement, we have to use a device which is able to measure a microwave reflection coefficient $\left(S_{11}\right)$ and a microwave-photonic transmission coefficient $\left(S_{21}\right)$. To reach this measurement, a Vector Network Analyser (VNA) is required. The experimental setup depends on the VCSEL structure (three different emitting wavelengths: 850nm, 1310nm and 1550nm) and the electrical access. For the LW-VCSEL, the VNA contains an optical rack able to measure directly the microwave-photonic $S_{21}$ coefficient. For the 850nm VCSEL, an optical fiber linked to a calibrated photodetector allows the $S_{21}$ measurement according to the experimental setup of Fig.20. 


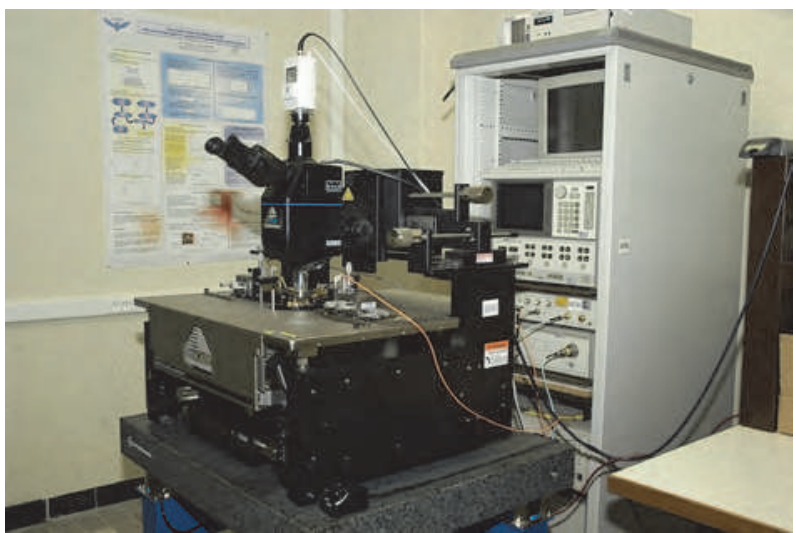

Fig. 19. RF probe station connected with a vector network analyzer

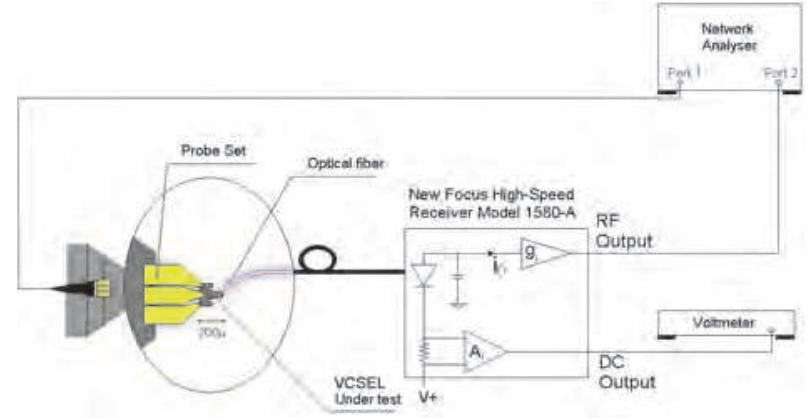

Fig. 20. Measurement of VCSEL response in opto-microwave domain
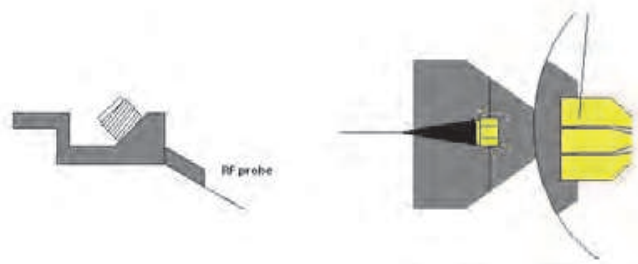

Fig. 21. RF probe

For the chip measurement, the VNA is coupled with a probe station as shown by Fig.19. The driving signal is brought by an RF probe as presented in Fig.21.

\subsection{Experimental results}

The experimental results presented in this section are achieved with the same experimental setup presented in the previous section. By thismeasurement technique, the influence of the electrical access is highlighted for two kind of VCSEL array topology. The TO packaging is excluded for two reasons: it doesn't allow the driving of only one VCSEL from an array 
and other extraction techniques have already been presented such as relative intensity noise measurement (Majewski \& Novak, 1991), subtraction procedure (Cartledge \& Srinivasan, 1997). So, we will focus on a VCSEL array with microstrip line electrical access and a VCSEL array with coplanar access. The model is validated by comparing the measurement and S-parameters simulation by implementing the equivalent circuit in the $A D S^{T M}$ software (RF simulation tool).

\subsubsection{Microstrip line electrical access}

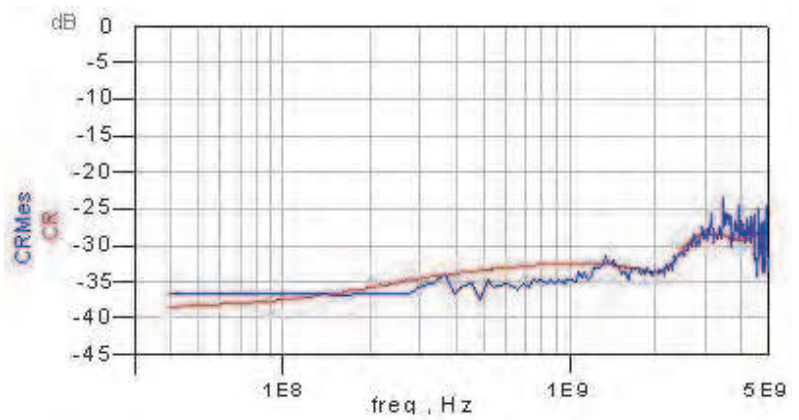

Fig. 22. Crosstalk measurement (blue curve) and simulation (red curve) of a VCSEL array with microstip access

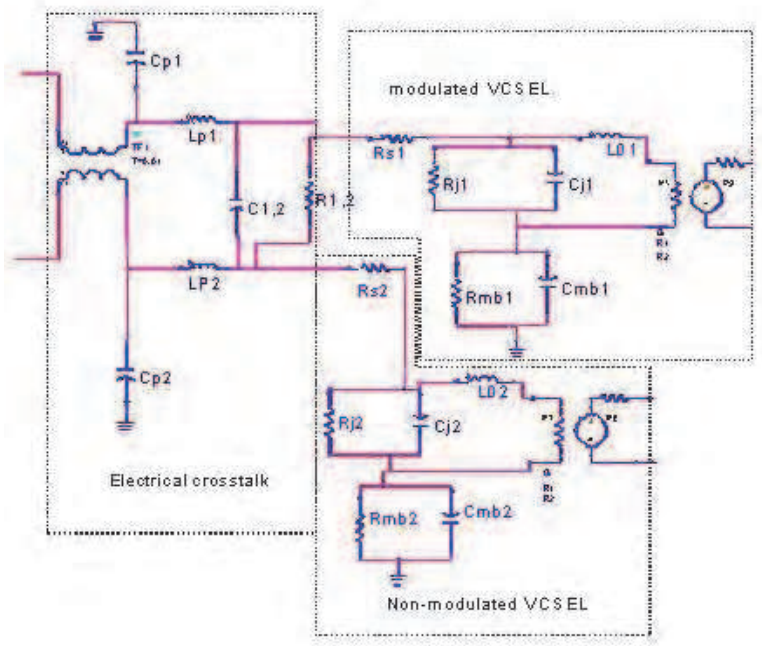

Fig. 23. Electrical equivalent circuit of two VCSELs including the crosstalk contribution

The measurement of S-parameters have been achieved on 850nm VCSEL arrays with microstrip line access. The microstrip line access requires the submount presented on Fig.13 to complete the RF tests and the integration in an optical subassembly. However this circuit involves parasitic effects clearly visible on the $S_{11}$ and $S_{21}$ measurement and a coupling 
between adjacent VCSEL. As we assume a negligible optical crosstalk, this coupling is the result of an impedance mismatch due to the wire bonding (Nakagawa et al., 2000). The RF modulation can circulate on the neighbouring wire by capacitive and inductive coupling. Through these couplings, carriers are injected in the adjacent laser involving a parasitic light emission. From the $S_{21}$ measurement, it is thus possible to get the crosstalk versus frequency. Fig.22, printing the crosstalk up to $5 \mathrm{GHz}$, is obtained by the difference between the $S_{21}$ of the non-driven VCSEL and the modulated VCSEL.

By using the VCSEL electrical equivalent circuit and by the available crosstalk model (represented by a mutual inductance, a parasitic inductance and capacitance), an electrical equivalent circuit of two VCSELs can be developed as shown in Fig.23. The simulation results are given by the red curve of Fig.22. The characterization and the modeling of this crosstalk is not significant below $1 \mathrm{GHz}$ (less than $-35 \mathrm{~dB}$ ) but it increases quickly above this frequency. The degradation of the $S_{21}$ measurement due to this coupling makes impossible the extraction of reliable value of the VCSEL intrinsic parameters.

\subsubsection{Coplanar access}
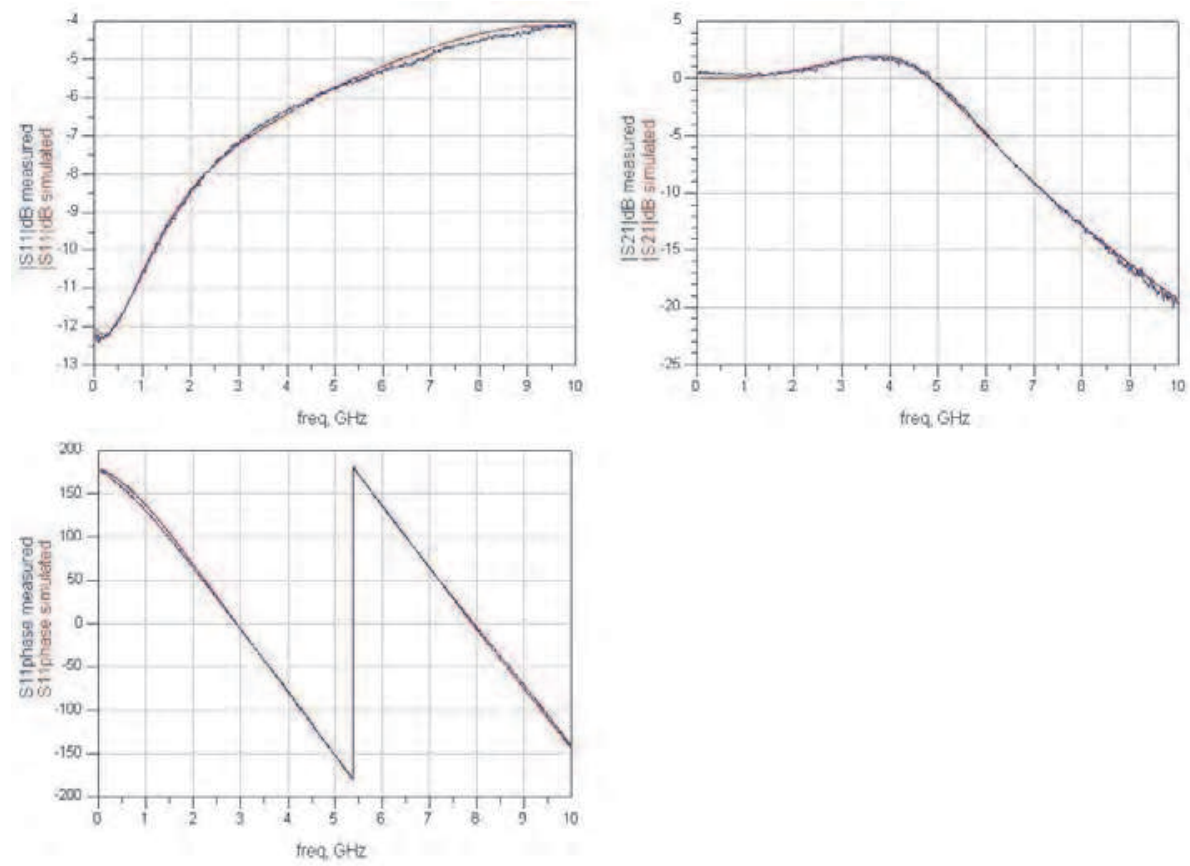

Fig. 24. Comparison between simulated and measured $S_{11}$ module and phase and $S_{21}$ module of a $850 \mathrm{~nm}$ VCSEL

The second kind of measurements is achieved on oxide confined 850nm VCSEL arrays with a coplanar access such as given by Fig.14. The measured scattering parameters of the VCSEL chip including the electrical access are so smouth (without numerical averaging of the VNA) that the extraction of the VCSEL cavity $S_{21}$ becomes available for the extraction of the equivalent circuit elements. According to the range of values of intrinsic parameters 
and by using the Equations 44,45, 47 and 48, a value of each intrinsic parameters can be fixed with a good agreement as it is shown in the comparison between the simulated $S_{21}$ (Fig.24, red curve) and the measured $S_{21}$ (Fig.24, blue curve). As the VCSEL tested are

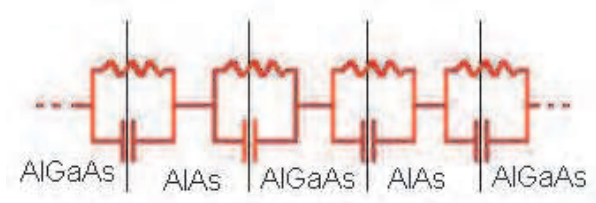

Fig. 25. RC distributed of DBR heterojunctions

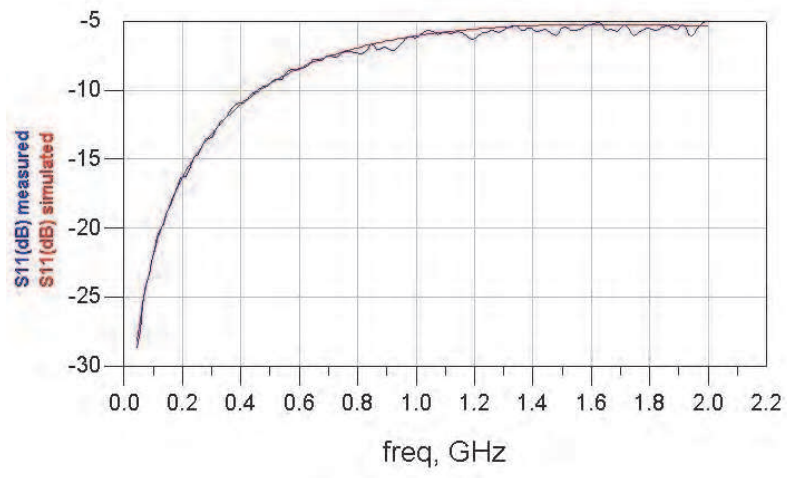

Fig. 26. Measured and simulated $S_{11}$ DBR with $10 \mu m$ ring contact

850nm oxide confined VCSEL, with a doped DBR, additional measurement can be achieved to extract the DBR equivalent circuit. As aforementioned, DBRs are constituted by a stack of heterojunctions. Each heterojunction implies RC parallel cell. Consequently, if we consider the whole DBR, we have as much resistances $\left(R_{i}\right)$ and capacitances $\left(C_{j}\right)$ as interfaces (Brusenbach et al., 1993). By considering that each interface of a DBR is identical, the electrical effects in a mirror is represented by an equivalent resistance $R_{m}$ and capacitance $C_{m}$ such as:

$$
\begin{gathered}
C_{m}=\frac{C_{i}}{2 n-1} \\
R_{m}=(2 n-1) \cdot R_{i}
\end{gathered}
$$

The top and bottom DBR having different doping and layer pair numbers, the equivalent

\begin{tabular}{l|l|l|l|l|l|l}
\hline $\begin{array}{l}\Phi \\
(\mu \mathrm{m})\end{array}$ & $\begin{array}{l}R_{m} \\
(\Omega)\end{array}$ & $\begin{array}{l}C_{m} \\
(\mathrm{pF})\end{array}$ & $\begin{array}{l}R_{\text {icalculated }} \\
(\Omega)\end{array}$ & $\begin{array}{l}C_{\text {icalculated }} \\
(\mathrm{pF})\end{array}$ & $\begin{array}{l}R_{i_{s} \text { imulated }} \\
(\Omega)\end{array}$ & $\begin{array}{l}C_{i_{s} \text { imulated }} \\
(\mathrm{pF})\end{array}$ \\
\hline & & & & & & \\
10 & 36 & 1.43 & 10.18 & 5.05 & 10.21 & 5.05 \\
12 & 20.8 & 3 & 5.88 & 10.6 & 5.88 & 10.6 \\
14 & 14.11 & 2.93 & 3.99 & 10.35 & 3.99 & 10.35 \\
\hline
\end{tabular}

Table 1. Comparison of the DBR resistances and capacitances 
resistances and capacitances are thus different for the two mirrors.

Indeed, DBR stacks have been tested to check the values of the capacitances and resistances. The $S_{11}$ of 8-p type DBR layer pairs grown by ULM Photonics GmbH (which provided VCSEL arrays). The test wafer contains different mesa with a ring contact on the top. Thus, the measurement is achieved by putting the signal pin of the coplanar probe on the ring contact and the ground pin on the substrate. As the geometry of the wafer hasn't a coplanar access, the measurement frequency range is limited to $2 \mathrm{GHz}$ (above this frequency the signal is to degraded to be exploited). By using the same approach than the preceeding one, the small signal equivalent circuit is implemented in $A D S^{T M}$ software and compared with the measurements. The characterization having been performed for different mesa diameters, the

\begin{tabular}{|c|c|c|}
\hline Parameters & Units & Values \\
\hline$A$ & $s^{-1}$ & {$\left[1.10^{8} ; 1,3.10^{8}\right]$} \\
\hline$B$ & $s^{-1}$ & {$\left[0,7.10^{-16} ; 1,8.10^{-16}\right] * V_{a c t}$} \\
\hline$N_{t r}$ & - & {$\left[0,83.10^{24} ; 4,4.10^{24}\right] * V_{a c t}$} \\
\hline$\tau_{p}$ & ps & {$[1 ; 6]$} \\
\hline$a$ & $m^{2}$ & {$\left[0,2 \cdot 10^{-20} ; 3,7 \cdot 10^{-20}\right]$} \\
\hline$V_{g r}$ & $m \cdot s^{-1}$ & {$\left[8,33.10^{7} ; 8,6.10^{7}\right]$} \\
\hline$\delta^{\prime}$ & - & {$[0,045 ; 0,06]$} \\
\hline$\beta$ & - & {$\left[10^{-5} ; 10^{-4}\right]$} \\
\hline$\eta_{i}$ & - & {$[0,6 ; 0,86]$} \\
\hline
\end{tabular}

Table 2. Range of $850 \mathrm{~nm}$ VCSEL intrinsic parameters

resistances and capacitances values can be calculated according to Equations 52 and 53 and the values for each diameters is presented in table 1. Fig.26 gives the comparison between the measurement and the simulation of $S_{11}$ module of $10 \mu \mathrm{m}$ diameter DBR. The agreement between the $S_{11}$ simulation and measurement is quite good and allows us to implement the $R_{m}$ and $C_{m}$ values into the VCSEL equivalent circuit.

From Equations 44, 45, 47, 48 and the values given by the table 2 for 850nm VCSEL, the intrinsic parameter values are extracted. $A, B, \beta, N_{t r}, \tau_{S}, a, v_{g r}, \eta_{i}$ and $\Gamma$ are obtained by optimization according to $R_{j}, C_{j}, L_{0}, R_{0}$ and the known VCSEL parameters $V_{T}, q, N_{w}, I_{0}, I_{t h}$, $V_{\text {act }}$. Thus for a $25 \mu \mathrm{m}$ oxide aperture VCSEL, we get the values:

$V_{T}=0.063 \mathrm{~V}, N_{w}=3, I_{0}=2 m A, I_{t h}=0.9 m A, \tau_{p}=6 p s, v_{g r}=8.5 \cdot 10^{7} \mathrm{~m} / \mathrm{s}, a=$ $1.76 \cdot 10^{-20} m^{2}, \beta=10^{4}, \Gamma=0.049, \eta_{i}=0.6, A=10^{8} \cdot \mathrm{s}^{-1}, B=88 \cdot \mathrm{s}^{-1}, N_{t r}=2.41$.

By the way of the Equation, the extracted values are:

- The threshold carrier number: $N_{t h}=3.04 \cdot 10^{6}$

- The photon number: $S=2.47 \cdot 10^{4}$

- The modal gain: $G=5.55 \cdot 10^{10}$

- The compression factor: $\epsilon=2.11 \cdot 10^{-6}$

The model gives then realistic results under certain conditions of VCSEL.

For the 1.55 $\mu \mathrm{m}$ VCSEL with a coplanar access, the same approache is used. The intracavity contacts avoid the contribution of the DBR into the electrical circuit. These wavelengths allow to extend the frequency range ut to $20 \mathrm{GHz}$ by using the calibrated optical rack with the VNA $\mathrm{HP} 8510 \mathrm{C}$. However, this frequency increase shows a $S_{21}$ response with a $-60 \mathrm{~dB} /$ decade slope in spite of the expected $-40 \mathrm{~dB} /$ decade. By knowing that a VCSEL has a $-40 \mathrm{~dB} /$ decade slope, the $-60 \mathrm{~dB} /$ decade slope is assumed to be induced by the electrical access topology (Fig.27). 


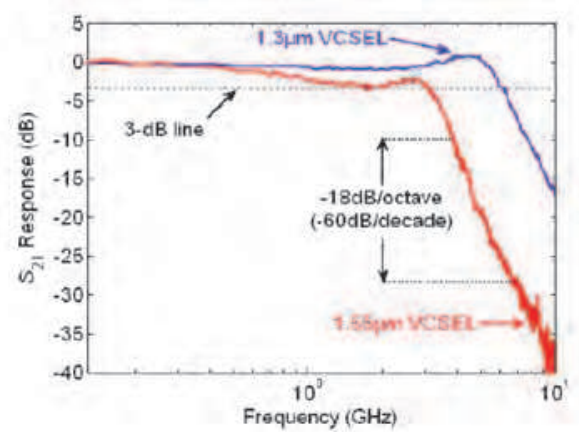

Fig. 27. Measured and extracted $S_{21}$ responses for a 1.3 $\mu \mathrm{m}$ VCSEL at a fixed bias current. The measurement takes into account the response of the electrical access as well as the cavity while the extracted curve shows the VCSEL cavity response only

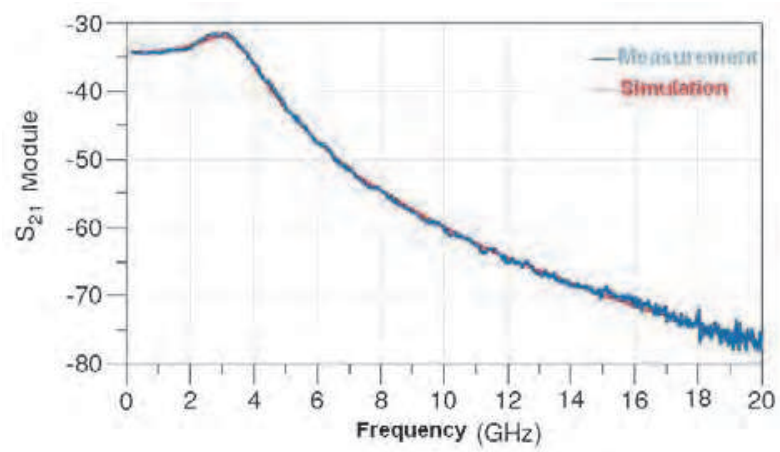

Fig. 28. Comparison between simulated and measured $S_{21}$ module of a $1.55 \mu m$ VCSEL

This effect has been modeled by using the tranfer matrix (T-matrix) formalism as presented by A. Bacou in (Bacou et al., 2009) and (Bacou et al., 2010). The method using the T-matrix consists in the splitting of the VCSEL chip into two cascaded subsystems representing the electrical access and the optical cavity, respectively. The equivalent electrical circuit defining the behavior of the electrical access is combined with the T-Matrix formalims to remove the parasitic contribution with the $S_{21}$ measurement response. The results allow to extract the $S_{21}$ of the VCSEL as shown by Fig.28 given a $-40 \mathrm{~dB} /$ Decade slope.

\section{Conclusion}

As it has been presented, the VCSEL technology is in current progress due to its wide advantages. The vertical emission allows a high integration level offering the possibility of $1 \mathrm{D}$ and 2D array. Due to the small size and the closeness of each VCSEL in an array, the possibility of electrical coupling on the electrical access is increased particularly for microwave frequency modulations. That's why the electrical access has a higher importance than it has for edge emitting laser diodes. A sight of the consequences of a mismatched electrical access has been presented here through a microwave-photonic study based on an electrical equivalent circuit model and the $S_{11}$ and $S_{21}$ parameters measurements. The results presented for the VCSEL 
array with a microstip-line electrical access shows that influence. Indeed, a coupling between the access lines involves an electrical crosstalk having repercussions on the emitted light by the VCSEL. The measurements achieved on a VCSEL array with a coplanar access bring the proof that a matched electrical access really improves the frequency response of the VCSEL array. Nevertheless, the matching is reached only for a certain frequency range. For wider frequency band of measurement, an increase of the $S_{21}$ slope has been observed. Even if the response of the VCSEL has been extracted, the electrical access design has to be improved for an impedance matching according to the frequency utilization. Thus this modeling an characterisation could further help in the VCSEL design and in the VCSEL integration.

\section{References}

Baba, T., Yogo, Y., Suzuki, K., Koyama, F. \& Iga, K. (1993). Near room temperature continuous wave lasing characteristics of gainasp/inp surface emitting laser, Electronics Letters 29(10): $913-914$.

Babic, D., Streubel, K., Mirin, R., Margalit, N., Bowers, J., Hu, E., Mars, D., Yang, L. \& Carey, K. (1995). Room-temperature continuous-wave operation of 1.54- mu;m vertical-cavity lasers, Photonics Technology Letters, IEEE 7(11): 1225 -1227.

Bacou, A., Hayat, A., Iakovlev, V., Syrbu, A., Rissons, A., Mollier, J.-C. \& Kapon, E. (2010). Electrical modeling of long-wavelength vcsels for intrinsic parameters extraction, Quantum Electronics, IEEE Journal of 46(3): 313 -322.

Bacou, A., Hayat, A., Rissons, A., Iakovlev, V., Syrbu, A., Mollier, J.-C. \& Kapon, E. (2009). Vcsel intrinsic response extraction using $\mathrm{t}$-matrix formalism, Photonics Technology Letters, IEEE.

Boucart, J., Starck, C., Gaborit, F., Plais, A., Bouche, N., Derouin, E., Goldstein, L., Fortin, C., Carpentier, D., Salet, P., Brillouet, F. \& Jacquet, J. (1999). 1-mW CW-RT monolithic VCSEL at $1.55 \mu \mathrm{m}$, IEEE Photonics Technology Letters 11(6): 629-631.

Brusenbach, P., Swirhun, S., Uchida, T., Kim, M. \& Parsons, C. (1993). Equivalent circuit for vertical cavity top surface emitting lasers, Electronics Letters 29(23): 2037 -2038.

Cartledge, J. \& Srinivasan, R. (1997). Extraction of dfb laser rate equation parameters for system simulation purposes, Lightwave Technology, Journal of 15(5): 852 -860.

Esaki, L. (1974). Long journey into tunneling, Proceedings of the IEEE 62(6): 825 - 831.

Ibaraki, A., Kawashima, K., Furusawa, K., Ishikawa, T. \& Yamaguchi, T. (1989). Buried heterostructure GaAs/GaAlAs distributed Bragg reflector surface emitting laser with very low threshold (5.2 $\mathrm{mA}$ ) under room temperature CW conditions, Japanese Journal of Applied Physics 28: L667+.

Iga, K., Koyama, F. \& Kinoshita, S. (1988). Surface emitting semiconductor lasers, Quantum Electronics, IEEE Journal of 24(9): $1845-1855$.

Jewell, J., Scherer, A., McCall, S., Lee, Y., Walker, S., Harbison, J. \& Florez, L. (1989). Low-threshold electrically pumped vertical-cavity surface-emitting microlasers, Electronics Letters 25(17): $1123-1124$.

Kapon, E., Sirbu, A., Iakovlev, V., Mereuta, A., Caliman, A. \& Suruceanu, G. (2009). Recent developments in long wavelength vcsels based on localized wafer fusion, Transparent Optical Networks, 2009. ICTON '09. 11th International Conference on.

Kopf, R. F., Schubert, E. F., Downey, S. W. \& Emerson, A. B. (1992). N- and p-type dopant profiles in distributed bragg reflector structures and their effect on resistance, Applied Physics Letters 61(15): $1820-1822$. 
Koyama, F. (2006). Recent advances of vcsel photonics, Journal Of Lightwave Technology Vol.24(No.12): 4502-4513.

Ly, K., Rissons, A., Quentel, F., Pez, M., Gambardella, E. \& Mollier, J.-C. (2008). Bidirectional link mock-up for avionic applications, IEEE Conference on Avionic FiberOptic (AVFOP), Publisher, San Diego, pp. xx-yy.

Majewski, M. \& Novak, D. (1991). Method for characterization of intrinsic and extrinsic components of semiconductor laser diode circuit model, Microwave and Guided Wave Letters, IEEE 1(9): $246-248$.

Nakagawa, S., Hu, S.-Y., Louderback, D. \& Coldren, L. (2000). Rf crosstalk in multiple-wavelength vertical-cavity surface-emitting laser arrays, Photonics Technology Letters, IEEE 12(6): $612-614$.

O'Brien, C., Majewski, M. \& Rakic, A. (2007). A critical comparison of high-speed vcsel characterization techniques, Lightwave Technology, Journal of 25(2): 597 -605.

Ortsiefer, M., Shau, R., Böhm, G., Köhler, F., Abstreiter, G. \& Amann, M.-C. (2000). Low-resistance inga(al)as tunnel junctions for long wavelength vertical-cavity surface-emitting lasers, Japanese Journal of Applied Physics 39(Part 1, No. 4A): $1727-1729$.

URL: http://jjap.jsap.jp/link?JJAP/39/1727/

Perchoux, J., Rissons, A. \& Mollier, J.-C. (2008). Multimode vcsel model for wide frequency-range rin simulation, Optics Communications 281(1): 162 - 169.

Rhew, K. H., Jeon, S. C., Lee, D. H., Yoo, B.-S. \& Yun, I. (2009). Reliability assessment of 1.55- $\mu m$ vertical cavity surface emitting lasers with tunnel junction using high-temperature aging tests, Microelectronics Reliability 49(1): $42-50$.

Rissons, A. \& Mollier, J.-C. (2009). Critical study of the vertical-cavity surface emitting laser electrical access for integrated optical sub-assembly, EMC Europ Workshop, Publisher, Athens, pp. xx-yy.

Rissons, A., Mollier, J., Toffano, Z., Destrez, A. \& Pez, M. M. (2003). Thermal and optoelectronic model of VCSEL arrays for short-range communication, in C. Lei \& S. P. Kilcoyne (ed.), Society of Photo-Optical Instrumentation Engineers (SPIE) Conference Series, Vol. 4994 of Society of Photo-Optical Instrumentation Engineers (SPIE) Conference Series, pp. 100-111.

Salet, P., Gaborit, F., Pagnod-Rossiaux, P., Plais, A., Derouin, E., Pasquier, J. \& Jacquet, J. (1997). Room-temperature pulsed operation of $1.3 \mathrm{mu}$;m vertical-cavity lasers including bottom ingaasp/inp multilayer bragg mirrors, Electronics Letters 33(24): 2048 -2049.

Scott, J., Geels, R., Corzine, S. \& Coldren, L. (1993). Modeling temperature effects and spatial hole burning to optimize vertical-cavity surface-emitting laser performance, Quantum Electronics, IEEE Journal of 29(5): 1295-1308.

Shau, R., Ortsiefer, M., Rosskopf, J., Boehm, G., Lauer, C., Maute, M. \& Amann, M.-C. (2004). Long-wavelength inp-based vcsels with buried tunnel junction: properties and applications, Vol. 5364, SPIE, pp. 1-15.

URL: http://link.aip.org/link/?PSI/5364/1/1

Soda, H., Motegi, Y. \& Iga, K. (1983). Gainasp/inp surface emitting injection lasers with short cavity length, Quantum Electronics, IEEE Journal of 19(6): 1035 - 1041.

Suematsu, Y. \& Iga, K. (2008). Semiconductor lasers in photonics, Lightwave Technology, Journal of .

Syrbu, A., Iakovlev, V., Suruceanu, G., Caliman, A., Rudra, A., Mircea, A., Mereuta, A., Tadeoni, S., Berseth, C.-A., Achtenhagen, M., Boucart, J. \& Kapon, E. (2004). 
1.55- mu;m optically pumped wafer-fused tunable vcsels with 32-nm tuning range, Photonics Technology Letters, IEEE 16(9): 1991 -1993.

Tucker, R. S. \& Pope, D. J. (1983). Microwave circuit models of semiconductor injection lasers, IEEE Transactions On Microwave Theory And Techniques Vol. 31(No. 3): 597-605.

Zhang, J.-P. \& Petermann, K. (1994). Beam propagation model for vertical-cavity surface-emitting lasers: threshold properties, Quantum Electronics, IEEE Journal of 30(7): $1529-1536$. 


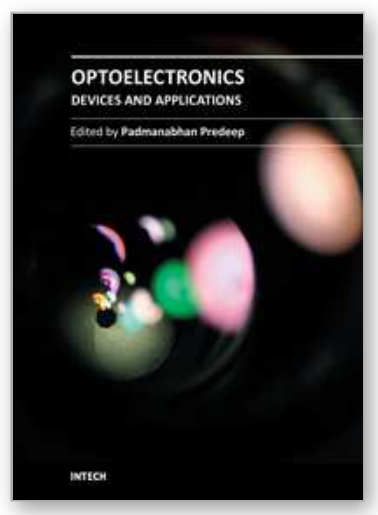

\author{
Optoelectronics - Devices and Applications \\ Edited by Prof. P. Predeep
}

ISBN 978-953-307-576-1

Hard cover, 630 pages

Publisher InTech

Published online 03, October, 2011

Published in print edition October, 2011

Optoelectronics - Devices and Applications is the second part of an edited anthology on the multifaced areas of optoelectronics by a selected group of authors including promising novices to experts in the field. Photonics and optoelectronics are making an impact multiple times as the semiconductor revolution made on the quality of our life. In telecommunication, entertainment devices, computational techniques, clean energy harvesting, medical instrumentation, materials and device characterization and scores of other areas of R\&D the science of optics and electronics get coupled by fine technology advances to make incredibly large strides. The technology of light has advanced to a stage where disciplines sans boundaries are finding it indispensable. New design concepts are fast emerging and being tested and applications developed in an unimaginable pace and speed. The wide spectrum of topics related to optoelectronics and photonics presented here is sure to make this collection of essays extremely useful to students and other stake holders in the field such as researchers and device designers.

\title{
How to reference
}

In order to correctly reference this scholarly work, feel free to copy and paste the following:

Angelique Rissons and Jean-Claude Mollier (2011). The Vertical-Cavity Surface Emitting Laser (VCSEL) and Electrical Access Contribution, Optoelectronics - Devices and Applications, Prof. P. Predeep (Ed.), ISBN: 978953-307-576-1, InTech, Available from: http://www.intechopen.com/books/optoelectronics-devices-andapplications/the-vertical-cavity-surface-emitting-laser-vcsel-and-electrical-access-contribution

\section{INTECH}

open science | open minds

\section{InTech Europe}

University Campus STeP Ri

Slavka Krautzeka 83/A

51000 Rijeka, Croatia

Phone: +385 (51) 770447

Fax: +385 (51) 686166

www.intechopen.com

\section{InTech China}

Unit 405, Office Block, Hotel Equatorial Shanghai

No.65, Yan An Road (West), Shanghai, 200040, China

中国上海市延安西路65号上海国际贵都大饭店办公楼 405 单元

Phone: +86-21-62489820

Fax: $+86-21-62489821$ 
(C) 2011 The Author(s). Licensee IntechOpen. This is an open access article distributed under the terms of the Creative Commons Attribution 3.0 License, which permits unrestricted use, distribution, and reproduction in any medium, provided the original work is properly cited. 